\title{
Meeting the expanded challenges of the cardiothoracic intensive care unit
}

Nevin M. Katz, MD

The cardiothoracic (CT) intensive care unit (ICU) increasingly represents an extension of the operating room. Over the years, the CT ICU has functioned to some extent as an operating room, for emergency procedures, including reopening of the chest for control of bleeding, and for small procedures, such as tracheostomy. However, with the increasing use of mechanical support devices, including extracorporeal membrane oxygenation and ventricular assist devices, and employment of "open chest protocols" for cases with coagulopathies and severe edema, CT surgeons and their operating teams are performing more procedures in the ICU. Hospitals have expanded the amount of support technology that is immediately available in these units.

Given the complexity level of the CT critical care that is now being performed, ICUs have the additional challenge of increasing monitoring capabilities, to optimize care and prevent organ injuries. Key to avoiding complications is the early detection of inadequate perfusion, to the brain, heart, kidneys, and intestinal tract. Metabolic markers are helpful, such as serum lactate levels; however, more specific monitoring is available, such as monitoring of cerebral perfusion with near-infrared spectroscopy.

The evolution of CT critical care has led to further expansion of CT critical care teams in many hospitals. ${ }^{2}$ Critical care nurses, surgeons, intensivists, anesthesiologists, perfusionists, and respiratory therapists have always been recognized as fundamental personnel on these teams. Many centers have come to recognize that nurse practitioners, physician assistants, and pharmacists have essential roles in providing timely optimal critical care, and actively participate on rounds and in "huddles" when sudden changes occur in a patient's condition. The multidisciplinary team now includes speech and language pathologists, physical therapists, and nutritionists, who all have important roles in patient evaluation and therapy.

The increasing challenges regarding severely ill patients who are in immediate danger of dying, or on maximal

\footnotetext{
From the Division of Cardiac Surgery, Johns Hopkins University, Baltimore, Md, and the Foundation for the Advancement of Cardiothoracic Surgical Care, McLean, Va. Disclosures: Author has nothing to disclose with regard to commercial support.

Received for publication June 3, 2015; accepted for publication June 4, 2015; available ahead of print July 14, 2015.

Address for reprints: Nevin M. Katz, MD, Johns Hopkins Hospital, 1800 Orleans St,

Suite 7107, Baltimore, MD 21287-4618 (E-mail: NevinKatz@aol.com).

J Thorac Cardiovasc Surg 2015;150:777-8

0022-5223/\$36.00

Copyright $(2015$ Published by Elsevier Inc. on behalf of The American Association for Thoracic Surgery

http://dx.doi.org/10.1016/j.jtcvs.2015.06.013
}

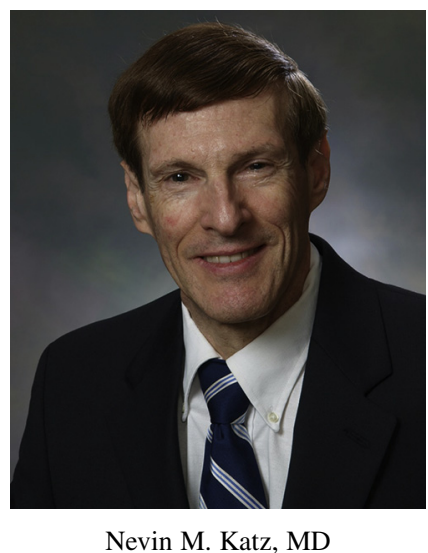

mechanical support, with no obvious "end of life," have led centers to create "supportive care" teams. They consist of staff with expertise in psychological, ethical, and financial issues for patients and families, as well as religious leaders. Unfortunately, these teams have been called "palliative care" teams by some centers. This term reflects an approach that is counter to the basic goal of the CT surgeon, which is to ultimately restore the patient to a meaningful life outside the hospital, and potentially "cure" the CT clinical issue that led to the patient's hospital admission. Clearly, "supportive care" is the appropriate term, and reflects the needs of many patients and families. This care is usually very much appreciated.

Communication among the multidisciplinary CT critical care team members continues to be a major challenge, as the numbers of both team members and complex situations they face increase. The $\mathrm{CT}$ surgeon continues to have a leadership role on the critical care team, even though the surgeon does not supervise the minute-to-minute care of the patient. ${ }^{3}$ As a specialty, CT surgery can embrace principles of the aviation industry referred to as "crew resource management." ${ }^{, 4}$ In addition to effective use of checklists, simulations, and debriefing procedures, crew resource management emphasizes the team concept, allowing any member of the team to raise an issue and suggest a change. An application of these principles was evident in the saving of all lives during the sudden ditching of US Airways flight 1549 into the Hudson River in New York, in 2009.

Most recently, federal legislation to create a value-based reimbursement system, as well as mandates to change surgical reimbursement from a 30-day to a 0-day global fee, has important implications. The Affordable Care Act has created a value-based system that focuses on optimal 
outcomes and reduction of complications and readmissions. ${ }^{5}$ To avoid penalties, medical centers need to refine their approach to the multidisciplinary specialty of CT critical care, which focuses on providing optimal care for optimal outcomes.

The expanded challenges highlight the importance of certification in critical care for CT surgeons. The American Board of Thoracic Surgery defines critical care as a core component of thoracic surgery and has specifically written 6 : "Thoracic Surgery encompasses the operative, perioperative, and surgical critical care of patients with acquired and congenital pathologic conditions within the chest."

Cardiothoracic surgeons have defined fundamental concepts in CT critical care. ${ }^{7}$ With the expansion of critical care training in thoracic surgery residencies, as well as the continuing medical education resources available, thoracic surgery as a specialty has established its credibility for certification. ${ }^{8}$ It is the CT surgeon who best understands those aspects of the physiology of cardiopulmonary bypass, as well as the cardiovascular, pulmonary, esophageal, and chest wall complications that are unique to thoracic surgery patients. In addition, thoracic surgeons perform procedures that are unique to CT critical care, such as insertion and removal of intra-aortic balloon pumps and other support devices.

Cardiothoracic surgeons will continue to draw on the expertise of medical specialists such as cardiologists, pulmonologists, nephrologists, and infectious disease specialists. However, the CT surgeon with expertise in CT critical care is in the best position to direct the critical care of CT patients. ${ }^{9}$ Clearly, if the American Board of
Thoracic Surgery can certify surgeons to perform adult and congenital cardiac surgery, pulmonary surgery, esophageal surgery, and chest surgery, it should be able to certify $\mathrm{CT}$ surgeons to provide the critical care needed by thoracic surgery patients. Clinical experience, continuing medical education courses, and examinations can form the criteria for certification.

Overall, the expanded challenges of CT critical care may require important changes in the organization and technology of ICU care at centers where CT surgery is performed. The CT surgeon has a leadership role on the multidisciplinary team that addresses these challenges.

\section{References}

1. Maldonado Y, Singh S, Taylor MA. Cerebral near-infrared spectroscopy in perioperative management of left ventricular assist device and extracorporeal membrane oxygenation patients. Curr Opin Anaesthesiol. 2014;27:81-8.

2. Katz NM. The evolution of cardiothoracic critical care. J Thorac Cardiovasc Surg. 2011;141:3-6

3. Katz NM. The emerging specialty of cardiothoracic surgical critical care: the leadership role of cardiothoracic surgeons on the multidisciplinary team. J Thorac Cardiovasc Surg. 2007;134:1109-11.

4. Grogan EL, Stiles RA, France DJ, Speroff T, Morris JA, Nixon B, et al. The impact of aviation-based teamwork training on the attitudes of health-care professionals. $J$ Am Coll Surg. 2004;199:843-8.

5. Lancaster E, Postel M, Satou N, Shemin R, Benharash P. Introspection into institutional database allows for focused quality improvement plan in cardiac surgery: example for a new global healthcare system. Am Surg. 2013;79:1040-4.

6. American Board of Thoracic Surgery. Booklet of information. Chigaco, IL: ABTS; January 2012.

7. Sherif HM. Developing a curriculum for cardiothoracic surgical critical care: impetus and goals. J Thorac Cardiovasc Surg. 2012;143:804-8.

8. Katz NM. It is time for certification in cardiothoracic critical care. J Thorac Cardiovasc Surg. 2013;145:1446-7.

9. Whitson BA, D'Cunha J. The thoracic surgical intensivist: the best critical care doctor for our thoracic surgical patients. Semin Thoracic Surg. 2011;23:12-3. 